\title{
Are antenatal interventions effective in improving multiple health behaviours among pregnant women? A systematic review protocol
}

Jenna L. Hollis ${ }^{1,2,3,4^{*}}$, Emma Doherty ${ }^{1,2,3,4}$, Julia Dray ${ }^{1,2,3,4}$, Danika Tremain ${ }^{1,4}$, Mandy Hunter ${ }^{5}$, Karen Takats ${ }^{5}$, Christopher M. Williams ${ }^{1,2,3,4}$, Henry Murray ${ }^{2,5}$, Craig E. Pennell ${ }^{2,4,5}$, Belinda Tully ${ }^{1,4}$, John Wiggers 1,2,3, Justine B. Daly ${ }^{1,4}$ and Melanie Kingsland ${ }^{1,2,3,4}$

\begin{abstract}
Background: Maternal behaviours in pregnancy associated with adverse pregnancy, birth and health outcomes include tobacco smoking, poor nutrition, alcohol consumption and low physical activity, collectively referred to as the SNAP risk factors. Due to the high prevalence, co-occurrence and possible interactive health effects of such health behaviours in pregnancy, antenatal interventions that support pregnant women to improve multiple SNAP behaviours have a greater potential impact on the health outcomes of women and their children than interventions addressing single behaviours. The objective of this review is to determine the effectiveness of interventions delivered as part of antenatal care that aim to improve multiple SNAP behaviours among pregnant women.

Methods: Seven electronic databases will be searched for potentially eligible studies. Eligible studies will include those where pregnant women are attending antenatal care. Studies that examine the effect of an intervention that addresses multiple SNAP behaviours ( $\geq 2$ behaviours) during pregnancy and are delivered or instigated through antenatal care in a healthcare senvice will be included. Systematic reviews of randomised controlled trials (RCTs), RCTs, cluster RCTs, stepped-wedge RCTs and nonrandomised control trials will be eligible. Studies that include a no-intervention control, wait-list control group, standard/usual care, or another active single behavioural intervention (e.g. addressing one behaviour only) will be considered. Two independent reviewers will conduct study screening, data extraction and risk of bias assessment. Discrepancies will be resolved by consensus or a third reviewer if required. A random effects model will be used to synthesise the results. Alternative synthesis methods will be investigated in instances where a meta-analysis is not appropriate, such as summarising effect estimates, combining $P$ values, vote counting based on direction of effect, or synthesis in narrative form.

(Continued on next page)
\end{abstract}

\footnotetext{
* Correspondence: jenna.hollis@health.nsw.gov.au

'Hunter New England Population Health, Hunter New England Local Health

District, Locked Bag 10, Wallsend, New South Wales 2287, Australia

${ }^{2} \mathrm{~S} c h o o l$ of Medicine and Public Health, The University of Newcastle, Callaghan, New South Wales, Australia

Full list of author information is available at the end of the article
}

(c) The Author(s). 2020 Open Access This article is licensed under a Creative Commons Attribution 4.0 International License, which permits use, sharing, adaptation, distribution and reproduction in any medium or format, as long as you give appropriate credit to the original author(s) and the source, provide a link to the Creative Commons licence, and indicate if changes were made. The images or other third party material in this article are included in the article's Creative Commons licence, unless indicated otherwise in a credit line to the material. If material is not included in the article's Creative Commons licence and your intended use is not permitted by statutory regulation or exceeds the permitted use, you will need to obtain permission directly from the copyright holder. To view a copy of this licence, visit http://creativecommons.org/licenses/by/4.0/ The Creative Commons Public Domain Dedication waiver (http://creativecommons.org/publicdomain/zero/1.0/) applies to the data made available in this article, unless otherwise stated in a credit line to the data. 
(Continued from previous page)

Discussion: The review will synthesise the evidence on the effect of interventions that address multiple SNAP behaviours in antenatal care and will help researchers, policy-makers and health services to develop and deliver best practice integrated models of antenatal care that have the potential to impact on both the short- and long-term health outcomes for women and their children.

\section{Systematic review registration: PROSPERO CRD42018095315}

Keywords: SNAP, Smoking, Nutrition, Diet, Alcohol, Physical activity, Behaviour change, Prenatal, Pregnancy, Maternity

\section{Introduction}

A woman's health behaviours during pregnancy have important implications for pregnancy and birth, as well as health outcomes for herself and her child both in the short term and across their life courses [1-6]. Tobacco smoking, poor nutrition, alcohol consumption and low physical activity, collectively referred to as the SNAP risk factors, are common maternal health behaviours associated with adverse health outcomes [7]. Pregnant women with multiple SNAP health behaviours are at even greater risk than those with single health behaviours, with evidence of a possible interactive effect of some health behaviours. For example, alcohol can impair the absorption and availability of maternal nutrient and energy intakes required for foetal development [8]. The cooccurrence of both smoking and alcohol consumption during pregnancy also increases the risk of preterm labour, low birth weight and growth restriction, beyond the individual effects of either smoking or alcohol [9]. Women with SNAP health behaviours in pregnancy are also more likely to continue to engage in these behaviours after pregnancy [10-13], increasing their risk of obesity [14] and chronic diseases including cardiovascular disease, type II diabetes, hypertension, dyslipidaemia, stroke and some cancers [15]. To address these risks, international $[16,17]$ and national guidelines $[18,19]$ recommend that pregnant women quit tobacco smoking, consume a diet that is adherent to food group recommendations, abstain from consuming alcohol and engage in 150 min of moderate physical activity each week.

Despite the risks associated with SNAP health behaviours and the guidelines advising pregnant women of recommended health behaviours, the prevalence of SNAP health behaviours in pregnancy is high. Internationally, studies have found that $10-30 \%$ of women smoke tobacco during pregnancy [20-24], 97-100\% do not meet all pregnancy food group recommendations $[25,26], 20-80 \%$ consume alcohol $[27,28]$ and $53-75 \%$ do not meet the recommended $150 \mathrm{~min}$ of moderate physical activity each week [29-31]. The clustering of SNAP health behaviours is well established in the general population [32, 33] and among pregnant women specifically [34-38]. In the Canadian Community Health Survey, pregnant women who smoked daily during pregnancy were 2.5 times more likely to have consumed alcohol in pregnancy compared with pregnant women who had never smoked in their life [35]. In the UK Avon Longitudinal Study of Parents and Children (ALSPAC) [39], consuming at least one unit of alcohol each day in the first trimester and consuming alcohol at high risk levels on single occasions (at least four units of alcohol in 1 day) in the first half of pregnancy were both associated with consuming a processed food dietary pattern, characterised by high intakes of processed meats, white bread and fried foods and low intakes of fruit and vegetables. The high prevalence and co-occurrence of adverse SNAP health behaviours in pregnant women supports the need for models of antenatal care that integrate best practice care for multiple health behaviours.

International [16] and national [17-19] antenatal care guidelines recommend that clinicians assess and support pregnant women to improve their SNAP health behaviours throughout the duration of their pregnancy. Health care services that provide antenatal care to women are opportune settings to engage and support pregnant women to improve their health behaviours during the antenatal period. Depending on their model of antenatal care, pregnant women may have an opportunity for regular contact with a range of primary health care clinicians including obstetricians, general practitioners, midwives and Aboriginal health workers and can be referred to other allied health and specialist services for additional behavioural support including dietitians, exercise physiologists, psychologists, smoking cessation counsellors and drug and alcohol specialists. Examining the effectiveness of interventions provided by, or instigated in, antenatal care could inform the delivery of routine integrated models of care for SNAP health behaviours.

Interventions that target co-occurring behavioural risks in pregnancy have potential for greater impact on a range of outcomes for women and their children than interventions addressing single behaviours [40], however research to date has focussed on the latter [40]. Previous reviews targeting co-occurring health behaviours in pregnancy have primarily focused on nutrition and physical activity as related to a specific health outcome, such as gestational weight gain [41]. There is limited evidence to guide health care providers on how to address multiple 
SNAP health behaviours [34] in integrated care pathways. To our knowledge, no systematic review has provided a synthesis of the field to examine the effect of interventions that address multiple SNAP behaviours on these health behaviours, or related health outcomes. The objective of this review is to determine the effectiveness of interventions delivered as part of antenatal care that aim to improve multiple SNAP behaviours among pregnant women. The secondary aim of this review is to determine if addressing multiple health behaviours results in greater behaviour change than targeting a single health behaviour.

\section{Methods}

The systematic review has been registered with the International Prospective Register of Systematic Reviews (PROSPERO; registration number CRD42018095315). The review protocol was reported in accordance with the Preferred Reporting Items for Systematic Reviews and Meta-Analyses Protocol (PRISMA-P) recommendations [42] (Additional file 1). The methods are consistent with those recommended by the Cochrane Handbook for Systematic Reviews of Interventions Version 6.0 [43].

\section{Outcomes}

The primary outcomes of the review are pregnant women's SNAP behaviour outcomes: tobacco smoking, nutrition intake, alcohol consumption and physical activity. The secondary outcomes are maternal and/or child health outcomes, and any unintended consequences or adverse outcomes of the intervention.

\section{Search strategy}

Electronic bibliographic databases will be searched for eligible peer reviewed literature, including: MEDLINE (Ovid), EMBASE (Ovid), PsycINFO (Ovid), Maternity and Infant Care (Ovid), Scopus, CINAHL (EBSCOhost) and Cochrane Library (Wiley). The reference lists of all included studies will be screened for other potentially eligible studies. The search strategy will be developed in consultation with a research librarian. There will be no restrictions on the length of the study follow-up period, country of origin or year of study. Only studies published in English will be eligible for consideration.

The strategy will include search terms for participant, intervention, comparator, outcome and study design. The database search term strategy for MEDLINE is described in Additional file 2. The search strategy will be adapted for other databases using appropriate syntax and terminology developed in consultation with a research librarian. The sensitivity of the study design search filter in detecting non-randomised study designs is unknown and it is possible that some such trials may have been missed.

\section{Eligibility criteria \\ Participants}

Studies of pregnant women receiving antenatal care at any time during pregnancy will be included. Studies of women in preconception or postnatal periods are not eligible for inclusion. Studies with interventions that span pregnancy and preconception or postnatal periods will be included only when intervention details and behavioural outcome data is outlined separately for the pregnancy period.

\section{Interventions}

Studies that include any intervention delivered during antenatal care, or instigated in antenatal care (e.g. referral to another health service for support), that aims to address multiple health behaviours (i.e. more than one SNAP behaviour) during pregnancy will be included. This includes all care provided or instigated in the antenatal care setting regardless of which health professional provided the care (e.g. antenatal clinician or health professional employed as a research personnel to provide care within the study). Antenatal settings can include primary care, hospital antenatal clinics, community antenatal clinics, home antenatal clinics, private obstetrics, midwifery care or allied health services. Studies with interventions that are in settings that are not usual health care providers to women during pregnancy, such as mass media health education campaigns, will be excluded. SNAP health behaviours include tobacco smoking, nutrition, alcohol consumption and physical activity. The intervention could include, but are not limited to, assessing pregnant women's health behaviour/s through validated and objective instruments, or self-reported behaviours; advising women on the health behaviour recommendations and providing education on the risks associated with adverse SNAP health behaviours during pregnancy; and providing behavioural support directly or offering a referral to other allied health and specialist services for additional support. Interventions only addressing sedentary behaviour will not be included in this review.

\section{Comparator}

Studies will be included that compare a multiple health behaviour intervention with no-intervention control, wait-list control, usual care, or another active intervention addressing one health behaviour (e.g. alcohol only).

\section{Outcomes}

Studies that include any measure of pregnant women's modifiable health behaviours, including tobacco smoking and/or cessation, nutrition intakes (e.g. reported as dietary intakes for energy, macronutrients or micronutrients; adherence to food or nutrient guidelines; or overall diet quality scores), alcohol consumption and/or abstinence and physical activity will be included. Outcome data can 
be collected via any data collection method (e.g. selfreport, observation, or objective measures) including audits of service or medical records such as patient pregnancy records.

\section{Types of studies}

Systematic review of randomised controlled trials (RCTs), RCTs, cluster RCTs, stepped-wedge RCTs (or staggered enrolment trials) and non-randomised controlled trials will be eligible for inclusion. Studies with designs without a parallel control group or another active intervention addressing one SNAP health behaviour (e.g. pre-post studies or historic control groups) will be excluded.

\section{Data collection and analysis \\ Study selection}

The titles and abstracts identified through the search strategy will be screened for eligibility by two independent reviewers using the eligibility criteria above. Studies that do not meet the criteria will be excluded. The full texts of all remaining studies will be reviewed by two independent reviewers, and the reason for exclusion will be recorded. Any discrepancies regarding study eligibility will be resolved through discussion and consensus between the two reviewers, or if required, consultation with a third reviewer. A reviewer will contact the study authors for clarification where there is insufficient information to determine study eligibility. If sufficient information is still unavailable, the study will be excluded from the review. Screening will be managed in Covidence (www.covidence.org).

\section{Data extraction}

Data from the eligible studies will be extracted by two independent reviewers. Data will be extracted using a standardised electronic form consistent with data collection items recommended by the Cochrane Handbook for Systematic Reviews of Interventions [43]. The form will be piloted prior to use. Any discrepancies in data extracted will be resolved by consensus between the two reviewers, or consultation with a third reviewer if required. Reviewers extracting data will not be blind to author or journal information.

The following information will be extracted:

- Study characteristics: authors (including contact details), article citation, date of publication, country of study, aim of study, dates intervention undertaken, participant characteristics (i.e. number of participants, age, body mass index, ethnicity, sociodemographic information, parity, clinical conditions, weeks' gestation), sample size, missing participants, study design, number of experimental groups, antenatal service setting (e.g. primary care, hospital antenatal clinics, home visit antenatal services or allied health) and information to assess risk of bias

- Intervention characteristics: SNAP behaviour change targeted, duration of intervention, number of contacts, theoretical underpinning of intervention and comparator, intervention content, mode of delivery (e.g. individual or group, in-person, online, telephone) and profession of intervention deliverer (e.g. midwife, obstetrician, dietitian, general practitioner, researcher)

- Outcomes: SNAP behaviour outcomes (smoking, nutrition, alcohol, physical activity) including definitions, maternal and/or infant health outcomes (i.e. health outcomes measured such as gestational weight gain, gestational diabetes, gestational hypertension, pre-eclampsia, preterm birth, low birth weight, macrosomia, foetal alcohol spectrum disorder and child and maternal obesity) including definitions, duration of follow-up, data collection method, name of tool, validity of measures used, scale of measure, number of participants per comparison group at each time point and effect size and measures of outcome variability

- Other: Key conclusions of the study authors, any unintended consequences or adverse outcomes, sources of funding and any potential conflicts of interest

\section{Assessment of risk of bias}

The risk of bias of the included studies will be assessed by two independent reviewers for both primary and secondary outcomes. The Cochrane Risk of Bias Tool, RoB2 [43], will be used to assess study characteristics for each RCT that is included in the review, including (i) bias from the randomisation process, (ii) bias from deviations from intended interventions, (iii) bias from missing outcome data, (iv) bias in the measurement of the outcome, and (v) bias in the selection of the reported results. An additional criteria will be used to assess risk of bias in cluster RCTs: (vi) bias arising from identification or recruitment of individual participants within clusters [43]. For included studies of non-randomised trial design, risk of bias will be assessed using the Risk Of Bias In Non-randomized Studies of Interventions (ROBINS-I) tool [44]. A consensus approach between the two reviewers will be used to resolve any assessment discrepancies, or a third reviewer with expertise in review methodology will be consulted.

\section{GRADE}

The GRADE approach [45] will be used to assess the overall quality of the evidence for the each of the four behavioural primary outcomes. Two independent reviewers will conduct the assessment, with discrepancies resolved through discussion and consensus between the 
two reviewers, or consultation with a third reviewer. Quality ratings ranging from 'very low' to 'high' will be assigned to each study.

\section{Measures of treatment effect}

Where possible, trial data will be combined and reported using meta-analyses using the standard estimation of (1) risk ratio (RR) and 95\% confidence intervals (CI) for dichotomous outcome variables, and (2) mean differences (MDs) or standardised mean differences (SMDs) and 95\% CIs for continuous outcome variables. We will use SMDs when studies report the same outcome and a comparable, but not identical, measure. In instances where a meta-analysis is not appropriate, alternative synthesis methods will be investigated as recommended by the Cochrane Handbook for Systematic Reviews of Interventions [43], such as summarising effect estimates, combining $P$ values, vote counting based on direction of effect, or synthesis in narrative form.

\section{Unit of analysis issues}

We expect to identify individually randomised and cluster-randomised studies for this review. We will examine cluster trials for unit of analysis errors. Where unit of analysis errors are identified, we will attempt to correct them prior to including the data in pooled analyses. For cluster design trials, individual level behaviour data adjusting for clusters using an intracluster correlation co-efficient (ICC) will be extracted. Study authors will be contacted to provide the ICCs for trials where the effects of clustering have not been reported. Where study authors are unable to provide ICCs, a mean ICC will be estimated from reported ICCs of included studies with similar behavioural outcome data and used to calculate effective sample sizes.

\section{Dealing with missing data}

Study outcome data analysed using the intention to treat (ITT) principle will be preferentially extracted over other outcome data (e.g. analysed using a completer's analysis), unless no ITT data is available. We will contact study authors to obtain missing data where it occurs. We will conduct sensitivity analysis excluding trials with high levels of missing outcome data (>30\%).

\section{Assessment of heterogeneity}

Clinical, methodological and statistical heterogeneity among studies will be considered when deciding if it is appropriate to perform a meta-analysis. Clinical heterogeneity will be firstly assessed to determine if the studies vary in intervention and outcomes reported. Variance in study design, outcome measure tools and risk of bias among the studies will be considered for methodological heterogeneity. Statistical heterogeneity of included studies will be examined using visual inspection of forest plots and statistically quantified by calculating the $I^{2}$ statistic [43]. An $I^{2}$ statistic of $>50 \%$ is considered to be substantial heterogeneity. We will perform subgroup analyses to identify the source of heterogeneity in such circumstances. Consensus will be sought between review authors regarding the appropriateness of a meta-analysis [43].

\section{Data synthesis}

Data from randomised and non-randomised study designs will be synthesised separately. Outcomes will be reported by intervention characteristics. Assuming the presence of heterogeneity, the primary and secondary outcomes will be combined in a meta-analysis using a random effects model (and the DerSimonian and Laird method to estimate the between-study variance) through the Review Manager 5 (RevMan 5) soLware (Review Manager 2014) and reported as a RR, MD, and SMD. Alternative synthesis methods will be conducted as recommended by the Cochrane Handbook for Systematic Reviews of Interventions [43] if studies cannot be combined in a meta-analysis.

Where studies report multiple of the same behavioural outcomes (e.g. for smoking cessation) using different data collection methods (e.g. self-report measures by women, health professional self-report, researcher observations or medical record audits), the outcome that is deemed to represent the most valid measure and/or for which the longest follow-up or most complete data is reported will be used. Only intervention and control groups that meet the eligibility criteria from multiple arm studies will be included in data synthesis. If a study contains multiple intervention or control arms that are all eligible for inclusion, a decision will be made to either (i) collapse all intervention and/or control arms into single pair wise comparisons or (ii) conduct bivariate analyses with all eligible arms included and adjust for the repeated inclusion of the same intervention and/or control arm.

\section{Assessment of reporting biases}

The methods and analyses of published studies will be compared to trial protocols and registers to identify instances of potential selective reporting. Funnel plots will be generated for each outcome to determine potential publication bias for meta-analyses including 10 or more studies.

\section{Sensitivity analysis}

Sensitivity analyses will be conducted for each of the SNAP behaviour outcomes where there are sufficient studies. This will be performed by removing studies with an overall high risk of bias to examine their impact on the effect estimate. 


\section{Discussion}

This systematic review will determine the effectiveness of interventions in antenatal care that aim to improve multiple SNAP health behaviours among pregnant women. The review will be of value to researchers, policy-makers and health services providing antenatal care. The findings will inform the development and delivery of effective integrated models of care for multiple health behaviours that have the potential to impact on short- and long-term health outcomes for women and children.

\section{Supplementary information}

Supplementary information accompanies this paper at https://doi.org/10. 1186/s13643-020-01453-z.

Additional file 1. PRISMA-P 2015 Checklist.

Additional file 2. Search Term Strategy.

\section{Abbreviations}

ALSPAC: Avon Longitudinal Study of Parents and Children; Cl: Confidence interval; ICC: Intracluster correlation co-efficient; ITT: Intention to treat; MDs: Mean differences; PRISMA-P: Preferred Reporting Items for Systematic Reviews and Meta-Analyses Protocol; PROSPERO: International Prospective Register of Systematic Reviews; RCTs: Randomised controlled trials; RR: Risk ratio; SMDs: Standardised mean differences; SNAP: Smoking, Nutrition, Alcohol, Physical activity

\section{Acknowledgements}

We would like to acknowledge Debbie Booth (Research Librarian) who assisted in developing the search strategy. JLH is supported by the Prevention Research Support Program, funded by the New South Wales Ministry of Health. MK has a Translating Research into Practice (TRIP) Fellowship funded by the National Health and Medical Research Council (APP1150476).

\section{Authors' contributions \\ $J \mathrm{LH}, J W, J B D$ and MK conceived the review and JLH led the development of the manuscript. $\mathrm{MH}, \mathrm{KT}, \mathrm{HM}, \mathrm{CEP}$ and $\mathrm{BT}$ provided expertise around antenatal care. JLH, ED, JD, DT, CMW, CEP, BT, JBD and MK provided expertise on the included risk factors. MK and ED provided guidance on the statistical analysis plan. All authors read and approved the final manuscript.}

\section{Funding}

Funding for this systematic review was received from the University of Newcastle Priority Research Centre for Health Behaviour. The funder played no role in developing the protocol.

\section{Availability of data and materials \\ Not applicable.}

\section{Ethics approval and consent to participate}

Not applicable.

\section{Consent for publication}

Not applicable.

\section{Competing interests}

The authors declare that they have no competing interests

\section{Author details}

${ }^{1}$ Hunter New England Population Health, Hunter New England Local Health District, Locked Bag 10, Wallsend, New South Wales 2287, Australia. ${ }^{2}$ School of Medicine and Public Health, The University of Newcastle, Callaghan, New South Wales, Australia. ${ }^{3}$ Priority Research Centre for Health Behaviour, The University of Newcastle, Callaghan, New South Wales, Australia. ${ }^{4}$ Hunter Medical Research Institute, New Lambton Heights, New South Wales,
Australia. ${ }^{5}$ Maternity and Gynaecology John Hunter Hospital, New Lambton Heights, New South Wales, Australia.

Received: 22 April 2020 Accepted: 13 August 2020

Published online: 02 September 2020

\section{References}

1. Bird AL, Grant CC, Bandara DK, Mohal J, Atatoa-Carr PE, Wise MR, et al. Maternal health in pregnancy and associations with adverse birth outcomes: evidence from growing up in New Zealand. Aust N Z J Obstet Gynaecol. 2017:57(1):16-24.

2. da Silva SG, Ricardo LI, Evenson KR, Hallal PC. Leisure-time physical activity in pregnancy and maternal-child health: a systematic teview and metaanalysis of randomized controlled trials and cohort studies. Sports Med. 2017:47(2):295-317.

3. Baidal JAW, Locks LM, Cheng ER, Blake-Lamb TL, Perkins ME, Taveras EM Risk factors for childhood obesity in the first 1,000 days: a systematic review. Am J Prev Med. 2016;50(6):761-79.

4. Banderali G, Martelli A, Landi M, Moretti F, Betti F, Radaelli G, et al. Short and long term health effects of parental tobacco smoking during pregnancy and lactation: a descriptive review. J Transl Med. 2015;13(1):327.

5. Patra J, Bakker R, Irving H, Jaddoe WW, Malini S, Rehm J. Dose-response relationship between alcohol consumption before and during pregnancy and the risks of low birthweight, preterm birth and small for gestational age (SGA) — a systematic review and meta-analyses. Br J Obstet Gynaecol. 2011; 118(12):1411-21.

6. Langley-Evans S. Nutrition in early life and the programming of adult disease: a review. J Hum Nutr Diet. 2015:28:1-14.

7. Harris M. Smoking, nutrition, alcohol and physical activity (SNAP): a population health guide to behavioural risk factors for general practices. Melbourne: Royal Australian College of General Practitioners; 2004.

8. Sebastiani G, Borrás-Novell C, Alsina Casanova M, Pascual Tutusaus M, Ferrero Martínez S, Gómez Roig M, et al. The effects of alcohol and drugs of abuse on maternal nutritional profile during pregnancy. Nutrients. 2018; 10(8):1008.

9. Odendaal HJ, Steyn DW, Elliott A, Burd L. Combined effects of cigarette smoking and alcohol consumption on perinatal outcome. Gynecol Obstet Investig. 2009;67(1):1-8.

10. Cooper S, Orton S, Leonardi-Bee J, Brotherton E, Vanderbloemen L, Bowker $\mathrm{K}$, et al. Smoking and quit attempts during pregnancy and postpartum: a longitudinal UK cohort. Br Med J Open. 2017;7(11):e018746.

11. Moran LJ, Sui Z, Cramp CS, Dodd JM. A decrease in diet quality occurs during pregnancy in overweight and obese women which is maintained post-partum. Int J Obes. 2012;37:704.

12. Tran NT, Najman JM, Hayatbakhsh R. Predictors of maternal drinking trajectories before and after pregnancy: evidence from a longitudinal study. Aust N Z J Obstet Gynaecol. 2015;55(2):123-30.

13. Pereira MA, Rifas-Shiman SL, Kleinman KP, Rich-Edwards JW, Peterson KE, Gillman MW. Predictors of change in physical activity during and after pregnancy: Project Viva. Am J Prev Med. 2007;32(4):312-9.

14. Mamun AA, Kinarivala M, O'Callaghan MJ, Williams GM, Najman JM, Callaway LK. Associations of excess weight gain during pregnancy with long-term maternal overweight and obesity: evidence from 21 y postpartum follow-up. Am J Clin Nutr. 2010;91(5):1336-41.

15. Australian Institute of Health and Welfare. Risk factors contributing to chronic disease. Canberra: Australian Institute of Health and Welfare; 2012.

16. World Health Organisation. WHO recommendations on antenatal care for a positive pregnancy experience. Geneva: World Health Organisation; 2016.

17. Abalos E, Chamillard M, Diaz V, Tuncalp Ö, Gülmezoglu A. Antenatal care for healthy pregnant women: a mapping of interventions from existing guidelines to inform the development of new WHO guidance on antenatal care. Br J Obstet Gynaecol. 2016;123(4):519-28.

18. Department of Health. Clinical practice guidelines: pregnancy care. Canberra: Australian Government Department of Health; 2018.

19. National Institute for Health and Care Excellence. Antenatal care for uncomplicated pregnancies. National Institute for Health and Care Excellence; 2008.

20. McCowan LM, Dekker GA Chan E, Stewart A Chappell LC, Hunter M et al. Spontaneous preterm birth and small for gestational age infants in women who stop smoking early in pregnancy: prospective cohort study. Br Med J. 2009;338:1081. 
21. Crozier SR, Robinson SM, Borland SE, Godfrey KM, Cooper C, Inskip HM, et al. Do women change their health behaviours in pregnancy? Findings from the Southampton Women's Survey. Paediatr Perinat Epidemiol. 2009; 23(5):446-53.

22. Owe KM, Nystad W, Bø K. Correlates of regular exercise during pregnancy: the Norwegian Mother and Child Cohort Study. Scand J Med Sci Sports. 2009;19(5):637-45

23. Caramaschi D, Taylor AE, Richmond RC, Havdahl KA, Golding J, Relton CL, et al. Maternal smoking during pregnancy and autism: using causal inference methods in a birth cohort study. Transl Psychiatry. 2018;8(1):262.

24. Fleisch AF, Rifas-Shiman SL, Rokoff LB, Hivert MF, Mantzoros CS, Oken E. Associations of maternal prenatal smoking with umbilical cord blood hormones: the Project Viva cohort. Metabolism. 2017;72:18-26.

25. Malek L, Umberger W, Makrides M, Zhou SJ. Adherence to the Australian dietary guidelines during pregnancy: evidence from a national study. Public Health Nutr. 2016;19(7):1155-63.

26. Morton SM, Grant CC, Wall CR, Carr PEA, Bandara DK, Schmidt JM, et al. Adherence to nutritional guidelines in pregnancy: evidence from the Growing Up in New Zealand birth cohort study. Public Health Nutr. 2014; 17(9):1919-29.

27. O'Keeffe LM, Kearney PM, McCarthy FP, Khashan AS, Greene RA, North RA, et al. Prevalence and predictors of alcohol use during pregnancy: findings from international multicentre cohort studies. Br Med J Open. 2015;5(7): e006323.

28. Strandberg-Larsen K, Poulsen G, Bech BH, Chatzi L, Cordier S, Dale MTG, et al. Association of light-to-moderate alcohol drinking in pregnancy with preterm birth and birth weight: elucidating bias by pooling data from nine European cohorts. Springer; 2017.

29. Lindqvist $M$, Lindkvist $M$, Eurenius $E$, Persson M, Ivarsson A, Mogren I. Leisure time physical activity among pregnant women and its associations with maternal characteristics and pregnancy outcomes. Sex Reprod Healthc. 2016;9:14-20.

30. Richardsen KR, Falk RS, Jenum AK, Mørkrid K, Martinsen EW, Ommundsen Y, et al. Predicting who fails to meet the physical activity guideline in pregnancy: a prospective study of objectively recorded physical activity in a population-based multi-ethnic cohort. BMC Pregnancy Childbirth. 2016; 16(1):186.

31. Australian Institute of Health and Welfare. Physical activity during pregnancy 2011-12. Canberra: Australian Institute of Health and Welfare; 2019. Contract No.: PHE 243.

32. Noble N, Paul C, Turon H, Oldmeadow C. Which modifiable health risk behaviours are related? A systematic review of the clustering of Smoking, Nutrition, Alcohol and Physical activity ('SNAP') health risk factors. Prev Med. 2015;81:16-41.

33. Meader N, King K, Moe-Byrne T, Wright K, Graham H, Petticrew M, et al. A systematic review on the clustering and co-occurrence of multiple risk behaviours. BMC Public Health. 2016;16(1):657.

34. Gilligan C, Sanson-Fisher R, Eades S, D'Este C, Kay-Lambkin F, Scheman S. Identifying pregnant women at risk of poor birth outcomes. J Obstet Gynaecol. 2009;29(3):181-7.

35. Lange S, Probst C, Quere M, Rehm J, Popova S. Alcohol use, smoking and their co-occurrence during pregnancy among Canadian women, 2003 to 2011/12. Addict Behav. 2015;50:102-9.

36. Cannon MJ, Dominique Y, O'Leary LA, Sniezek JE, Floyd RL. Characteristics and behaviors of mothers who have a child with fetal alcohol syndrome. Neurotoxicol Teratol. 2012;34(1):90-5.

37. Cooper $\mathrm{DL}$, Petherick ES, Wright J. Lifestyle related risk factors in a multiethnic cohort of pregnant women: preliminary results from the Born in Bradford study. Public Health. 2013;127(11):1034-7.

38. Doyle I-M, Borrmann B, Grosser A, Razum O, Spallek J. Determinants of dietary patterns and diet quality during pregnancy: a systematic review with narrative synthesis. Public Health Nutr. 2017;20(6):1009-28.

39. Coathup V, Northstone K, Gray R, Wheeler S, Smith L. Dietary patterns and alcohol consumption during pregnancy: secondary analysis of Avon Longitudinal Study of Parents and Children. Alcohol Clin Exp Res. 2017;41(6):1120-8.

40. Prochaska JJ, Prochaska JO. A review of multiple health behavior change interventions for primary prevention. Am J Lifestyle Med. 2011;5(3):208-21.

41. Yeo S, Walker JS, Caughey MC, Ferraro AM, Asafu-Adjei JK. What characteristics of nutrition and physical activity interventions are key to effectively reducing weight gain in obese or overweight pregnant women? A systematic review and meta-analysis. Obes Rev. 2017;18(4):385-99.
42. Moher D, Shamseer L, Clarke M, Ghersi D, Liberati A, Petticrew M, et al. Preferred reporting items for systematic review and meta-analysis protocols (PRISMA-P) 2015 statement. Syst Rev. 2015;4(1):1.

43. Higgins JPT, Thomas J, Chandler J, Cumpston M, Li T, Page MJ, et al. Cochrane Handbook for Systematic Reviews of Interventions version 6.0 (updated July 2019): Cochrane; 2019 [Available from: www.training. cochrane.org/handbook.

44. Sterne JA, Hernán MA, Reeves BC, Savović J, Berkman ND, Viswanathan M, et al. ROBINS-l: a tool for assessing risk of bias in non-randomised studies of interventions. BMJ. 2016;355:i4919.

45. Guyatt G, Oxman AD, Akl EA, Kunz R, Vist G, Brozek J, et al. GRADE guidelines: 1. Introduction-GRADE evidence profiles and summary of findings tables. J Clin Epidemiol. 2011;64(4):383-94.

\section{Publisher's Note}

Springer Nature remains neutral with regard to jurisdictional claims in published maps and institutional affiliations.

Ready to submit your research? Choose BMC and benefit from:

- fast, convenient online submission

- thorough peer review by experienced researchers in your field

- rapid publication on acceptance

- support for research data, including large and complex data types

- gold Open Access which fosters wider collaboration and increased citations

- maximum visibility for your research: over $100 \mathrm{M}$ website views per year

At BMC, research is always in progress.

Learn more biomedcentral.com/submissions 